Grand Valley State University

ScholarWorks@GVSU

Fall 1998

\title{
Continuous Quality Improvement, Total Quality Management, and Reengineering: One Hospital's Continuous Quality Improvement Journey
}

Donald Klein

Grand Valley State University

Jaideep Motwani

Grand Valley State University, motwanij@gvsu.edu

Beth Cole

Midwestern University - Glendale

Follow this and additional works at: https://scholarworks.gvsu.edu/mgt_articles

Part of the Business Commons

\section{ScholarWorks Citation}

Klein, Donald; Motwani, Jaideep; and Cole, Beth, "Continuous Quality Improvement, Total Quality Management, and Reengineering: One Hospital's Continuous Quality Improvement Journey" (1998). Peer Reviewed Articles. 6.

https://scholarworks.gvsu.edu/mgt_articles/6

This Article is brought to you for free and open access by the Management Department at ScholarWorks@GVSU. It has been accepted for inclusion in Peer Reviewed Articles by an authorized administrator of ScholarWorks@GVSU.

For more information, please contact scholarworks@gvsu.edu. 


\title{
Case Study
}

\section{Contimuous Quality Improvement, Total Quality Management, and Reengineering: One Hospital's Continuous Quality} Improvement Journey

\author{
Domald \\ *Seidman School of Business, Grand Valley State University, Grand Rapids, Michigan 49504, and $\ddagger$ Development Office, \\ Midwestern University, Glendale, Arizona 85308
}

In recent years, there has been significanty increasing interest in the application of continumous quaphy improvement (CQI) and total quality mangement (TQM) in the health care arena. This case analysis is designed 10 idertify and assess the strotegies and processes that led to the successful inplenentation of CQI in the Emergency Care Cemter at St. Mary's Hospitat in Grand Rapids, MI.

EDITORIAL COMMENT: In addition to this case study being presented as another quality assurance success story, it is offered to highlight its value as an effective model for combining accepted management techniques, ie, continuous qualily improvement and reengineering. The two are perceived by many to be mutually exclusive or, in the case of the latter, inappropriate for the health care arena. Also, this case demonstrates how the two techniques can be integrated to release a log jam which was a potential risk to the otherwise successful quality initiative. Further, it highlights the intriguing nature of quality management-a specialty which requires creative, forward thinking people who are bold enough to explore new ground in their quest for quality in health care. This quest embodies attention to form and function, process, and practice.

† To whom correspondence should be addressed at Seidman School of Business, Grand Valley State University, 301 West Fulton, \#510, Grand Rapids, MI 49504.

$\S$ Served as Director of Clinical Services at St. Mary's Health Services when the project was undertaken.
Finally, this positive management result ultimately constitutes a benefit for practitioners and contributes to optimum outcomes for patients. A win-win-win scenario.-Beverly Carpenter-Mason, Ph.D., P.N.P., R.N., Case Study Editor.

\section{MNTROOUCTION}

A survey of 781 hospital chief executive officer (CEO) respondents showed that $58.5 \%$ were presently implementing a total quality management (TQM) or continuous quality improvement (CQI) program; $84.6 \%$ of those who were not were planning on doing so in the next fiscal years (1). By adopting the concepts of TQM or CQI, a health care institution can move from an inspectionoriented quality improvement system to one that orients itself to a systematic transformation of an organizational culture through a roll-out plan involving customer focus, key-process monitoring, data-driven tools and techniques, and team empowerment (2).

This is a report of the findings of a case study analysis of the implementation of CQI at St. Mary's Hospital in Grand Rapids, MI. An in-depth, detailed investigation was conducted by the researchers to identify and assess the ingredients that lead to the successful implementation of CQI programs in the Emergency Care Center (ECC) of the hospital. The field work was undertaken from January to November 1996. 


\section{DESCRIPTVE BACKGROIND OF THE HOSPITAL}

Saint Mary's Hospital, founded in 1893, is owned by Mercy Health Services, an outreach of the Sisters of Mercy, Regional Community of Detroit, MI. The hospital is a 300-bed, full service, acute care medical center and teaching hospital serving Grand Rapids and surrounding cities. Since its beginnings, the hospital has provided emergency care, specializing in penetrating injuries. The community served by Saint Mary's Hospital includes Grand Rapids, the second largest and fastest growing city in Michigan. Grand Rapids and its surrounding suburbs comprise a population of more than 300,000 .

\section{MPLEMENTATION OF COI AT ST. MARY'S}

\section{Rationale}

A new management team was installed in December 1993 to guide and manage St. Mary's. This team quickly recognized that the culture had to be changed if the hospital was to succeed, due to the demands being placed on hospitals by the federal government and the general public to rapidly change practices and dramatically lower costs. The hospital was also cognizant of the need to decrease the time it was taking to process a patient through the ECC, from arrival time to disposition, particularly for patients who were being admitted. Consequently, it was decided to focus on the ECC first, using it to demonstrate teaching $\mathrm{CQI}$ techniques while applying them to specific issues in a specific arena.

\section{Methodology}

To facilitate the teaching of CQI techniques, the corporate arm of St. Mary's, Mercy Health Services, hired an expert, in CQI to assist its various hospitals. This individual came, taught $\mathrm{CQI}$ techniques, and started to work with the ECC staff using data from a previous operations management study. The initial information available to the team was data collected on the amount of time patients spent in the ECC on various segments of the process.

\section{Establishment of the Focus Team}

The hospital president asked the Director of Critical Care Services to be the facilitator of the emergency department's focus team along with one of the nurse man- agers from the emergency department. Because they were the most familiar with the individuals' skills and abilities, the facilitator and the nurse manager then selected the first multidisciplinary focus team. The first team numbered 19. To avoid having any one specialty feel it was not represented in this new process, every personnel category in the ECC had at least one representative. Hindsight indicates that the team was about three times larger then necessary. Size and varying opinions of what to do and how to do it initially slowed the team's efforts. This was not surprising, given the professional background and status of key players. St. Mary's learned a lot about the composition and size of study teams from this experience; and over time the team evolved into smaller, more workable units.

\section{Learning Approach}

Initially, this large team thought it was going to look at the whole process of the ECC-from patient entry to exit. The team started a flowchart on every process that occurred. In a short time, the team members had flowcharts wrapping around the four walls of the meeting room; hence, discovering they had "bitten off more then they could chew." Consequently, the team used brainstorming and multivoting techniques to hone in on what areas were thought to be the key causes for time delays in processing patients in and out of the emergency department. The corporate CQI expert acted as a facilitator instead of a formal trainer. She spent time teaching CQI techniques, after which the focus team would immediately apply the concepts to data that had been collected.

\section{Identification of Key Time Delay Causes}

The first key cause of time delay was transporting obstetrics (OB) patients to the $O B$ floor. Mothers who came in to deliver their babies had to go through the ECC and be transported by wheelchair to the 9 th floor. Investigation indicated that the only reason they came to the ECC was because it was open $24 \mathrm{hr}$ a day. No one ever challenged this policy. The only thing emergency room personnel did was transport the mother in a wheelchair to the OB floor. The staff perceived that this took a significant amount of time away from providing patient care in the emergency room; and it was also costly. Therefore a team was organized to study the issue.

A second team was formed to study the availability of supplies and equipment in the ECC. The initial team judged that this also caused a major delay in terms of 
getting patients processed through the $\mathbb{E C C}$. The medical personnel could not always quickly find the supplies and/or the equipment needed. Consequently, they had to search the ECC or other areas of the hospital for the appropriate supplies and equipment.

The collected data also revealed a third cause of lengthy delay in the ECC due to the time required for a patient to be admitted to a unit in the hospital from the ECC. A team also was appointed to investigate this perception.

The fourth cause of time delay focus was triage and registration. There was a perception that there was a shortage of registration clerks, leading to lengthy delays in registering patients to be seen by an ECC physician.

Rather than have a large team tackle these four time delay causes one at a time, the team of 19 was split into four smaller teams. Each team developed an opportunity statement for its assigned issue.

\section{Team Reporting Process}

In the beginning, each of the four new teams made monthly progress reports to the original large group which was now known as the ECC Express. This lasted approximately 6 months. After which the large group realized that reporting back was not the most efficient use of the ECC Express.

It was decided that each small team would pexiodically report to a specific senior leadership member of each of the hospital's several core strategy teams. Each of the four teams would provide written periodic reports as well as a verbal report to the whole core strategy team from time to time. This was of value not only for communication but also assured the teams' directions were consistent with the overall strategy of the hospital. The presentations basically focused on the steps of the PlanDo-Check-Act (PDCA) cycle (see Fig. 1).

\section{FOLLOWUP DETAL}

\section{OB Pathents 109 9h Floor}

The team researching the time it takes to get an $\mathrm{OB}$ patient from ECC to the 9th floor started by reviewing St. Mary's reasons for its current policy. Inquiries were made and discussions held with the $\mathrm{OB}$ floor and $\mathrm{ECC}$ personnel to explore how $O B$ patients could be better served. The team addressing these questions adhered to CQI principles and asked both sides to this continuous improvement opportunity, what are our alternatives? This was a real learning experience for all involved. Finally, staff chose to sit down with each other and learn to negotiate change and look at St. Mary's systems as a

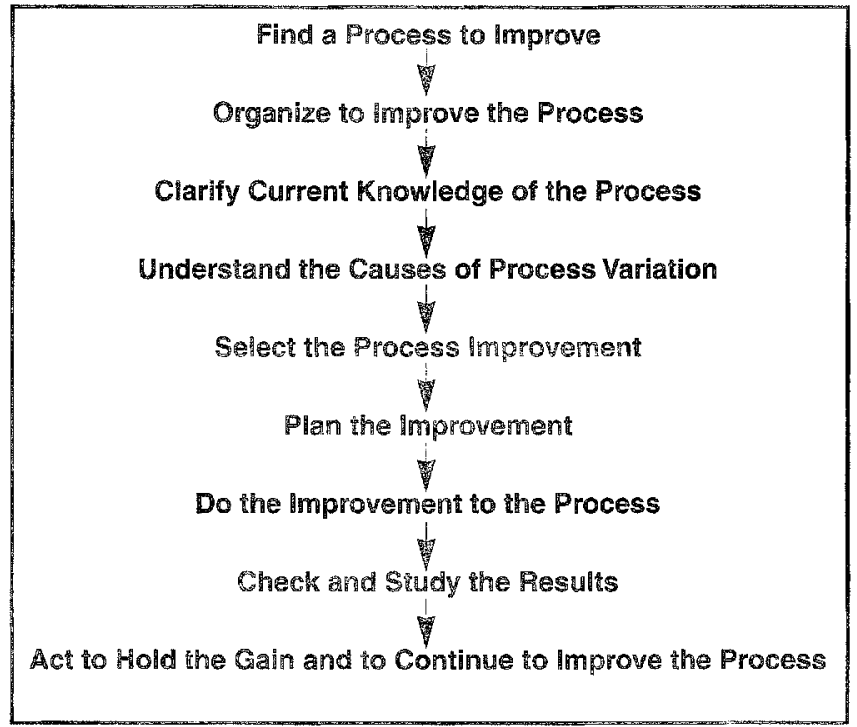

Fig. 1. Overview of the focus-PDCA approach used at St. Mary's.

synchronized whole. The emphasis was on customer satisfaction and optimum results; not territorial responsibility issues.

The first thing ECC and OB personnel did was actually study the cost of transferring patients to the OB floor. How much time was actually spent doing this? The staff's perception was that an inordinate amount of time was spent. However, results showed the cost actually was very small in terms of registered nurse time.

The second thing that occurred was the realization that the policy should be changed. More often than not a person about to deliver a baby would prefer to go directly to the $\mathrm{OB}$ floor. The two units decided that all $\mathrm{OB}$ patients would still enter the hospital through the ECC because it is open 24 hr a day. Upon entry OB patients would be asked if they felt comfortable to independently go up to the 9 th floor. Directions were given to the $\mathrm{OB}$ unit to each patient. Follow-up patient satisfaction surveys have indicated strong positive feedback.

Even though this first step in the use of CQI problem solving did not cost or save significant dollars, it did provide an early success. It increased customer satisfaction and staff morale, improved the working relationship between two units, enlightened people about how to negotiate with each other, and gave CQI additional credibility, momentum, and more ready acceptance.

\section{Registration Time}

A second CQI team studied the perception that there was a large time delay between registering patients and 
getting them into a bed in the ECC. There was continuous lamentation about how short-staffed registration was and that this was the reason for the long length of time to deliver a person to the ECC.

This CQI team focused on the patients that were not admitted to the hospital after visiting the $\mathrm{ECC}$. Once again, by going through the PDCA cycle and collecting and studying data, the team was better able to focus on what it should be doing. The team found that the infamous perception gap prevailed again. The ECC staff perceived that the laboratory had very poor turn around times. It was thought that when a specimen was sent to the laboratory it took $45 \mathrm{~min}$ to $1 \mathrm{hr}$ to get the results sent to the ECC. The expected time was 20-30 min. Consequently, the team focused on this aspect. Data were collected and inquiries made of the laboratory. Data showed that it was taking $45-60 \mathrm{~min}$ from the time a test was ordered for the results to get back to the ECC.

When the whole process was broken down it was found that it took the ECC $30 \mathrm{~min}$ to get the specimen to the laboratory. Half of the $60 \mathrm{~min}$ was contained in the ECC itself! This was an eye opener for ECC personnel. The long time delay was within their own control. Perceptions started to change once the facts were known. Edwards Deming again would say "know the facts."

The ECC was asked to develop a consistent process for collecting specimens and getting them to the laboratory. People in the ECC agreed it was a good idea and were very cooperative once they realized the facts. Without the CQI process and the data, this never would have happened.

Results to date are a reduction of laboratory draw/order times from an average of $14 \mathrm{~min}$ to $9 \mathrm{~min}$; the actual draw time from $8 \mathrm{~min}$ to $6 \mathrm{~min}$. The acceptance of a standardized process in itself is considered a success; without factoring in the reduction of time. This is another example of success of the CQI approach helping to change organizational culture to one of more cooperation, understanding, and some definitively measurable results.

This team is now looking at an area in the ECC called the Fast Track. The Fast Track is for patients with minor medical problems such as a sore throat, cold, or those requiring an $\mathrm{X}$-ray for a sprain or strain that does not require intensive resources. How can St. Mary's serve these types of patients faster?

\section{Supplies and Equipment}

A third CQI team worked on the availability of supplies and equipment. Too many times ECC staff had to scurry around trying to locate a needed item. One of the first discoveries of the team was that there were several systems to obtain supplies and equipment. The team worked with the materials management staff to understand what was occurring. One of the first facts recognized was there were different charge staff ordering on all three work shifts and the staff were being rotated. Different staff were ordering the same items and were not communicating the status of items ordered. Consequently, there was duplication of orders, stock outs, and overstocks regularly. Communication had to be enhanced between everyone ordering.

The team suggested that specific persons on each shift be the only ones to order supplies and equipment and that they be instructed to investigate any backorders before ordering. A simple system of how to process future orders was implemented. A commercial system was put in place for medical supplies and prescriptions. Each authorized person was given a code name and a personal identification number. The proprietary equipment was rented and interfaced with the billing and inventory system. Low use, high cost items were placed in the system machine for control and availability of use purposes. This system can also trace the items used directly to the patient's account for billing purposes; one less item of overhead to be allocated.

The team also looked at the inventory and use of sutures. By looking at the data, the team was able to eliminate some sutures as well as lower the inventory levels of the remaining sutures. It also invited bids on sutures and was able to save over $\$ 8,000$ the first year. Once again things are simplified, cost is reduced, and there is less staff frustration. Another CQI success story. This team is now investigating the many forms handled by the ECC as well as the many and various places they are required to be used.

\section{Adnoluting a Patient}

The fourth CQI team, which had the most members, also had the potential to affect the organization the most. They investigated the process of admitting a patient to the hospital from the ECC. It took a very long time for the team to flowchart and understand the process. The many variables involved made it difficult to capture what really occurred.

It was eventually determined that it took an average of $247 \mathrm{~min}$ (or a little over $4 \mathrm{hr}$ ) to admit a patient to a hospital unit from the time patients arrived in the ECC to the time they were in bed. The team decided this needed to be refined into a smaller segment of process time. The team focused on the time the decision was made in the ECC to admit, until the person was in a bed. It was determined that it was taking on average $90 \mathrm{~min}$ 
after the ECC decided to admit a patient until that patient was in bed.

\section{Results}

After applying the PDCA cycle several times the team felt it had not accomplished a significant change because of the size and complexity of the process. The team recognized there were many opportunities for improvements, but it would take a long time at the pace the team was proceeding. Senior leadership at St. Mary's suggested, after listening to team reports over several months, that perhaps the team should consider reengineering the process rather than expect CQI to fix an obviously broken component.

\section{REENGINEERING}

St. Mary's engaged the same firm that had worked with the team teaching $\mathrm{CQI}$ to teach the team reengineering. The consultant assigned to St. Mary's worked with the team as it learned reengineering. It was tedious and excruciating work because of the level of detail. Every facet of the process was flowcharted and analyzed for necessity, key quality characteristics, critical or noncritical nature, and value-added purposes. Each key quality characteristic was measured as to its relationship, if any, to necessity and customer needs. Were the relationships strong, weak, or nonexistent?

\section{Practice Sessions}

Creativity exercises were also used to generate a framework for the new process. The team had to assume and develop what the system would be in a perfect world without constraints of any kind. Hypothetical letters were written to the CEO as to how the new system would work, what its barriers were, and what the customers (patients) were saying about it. Another group assumed the role of the ECC director and described the new process and how it worked. These creative exercises helped to develop the open mindedness that ultimately led to the evolution of an improved process.

\section{On-going Implementation}

The team suggested that instead of waiting $30 \mathrm{~min}$ after the patient gets into a bed to request medical information, that patients medical records be requested at the time they register. This way the record would be available to the physician when entering the patient's room.
To do that, St. Mary's needed some means of ascertaining whether the patients had previously been admitted/seen. The team realized patient cooperation was critical to success. St. Mary's plans to give patients a card that includes their medical record number and other key medical information. Each time patients return to St. Mary's, they are asked to bring the card with them so their care needs can be expedited. The cards have been printed and a pilot project is now underway. Patient compliance with the request is most necessary. The team is optimistic that with continuous education and time, the card will lessen the time to get patients from the ECC to their beds.

The team also has recommended that a patient's case manager, if applicable, be listed on the hospital data information system. When patients register, their case manager's name is placed on the face sheet. The medical staff can contact the case manager directly for further information.

These suggestions will facilitate downstream patient care. Basically, the ECC physician will be gathering clinical information to make the decision to admit the patient. To facilitate this, an additional computer terminal has been placed in the ECC, so it is more convenient for the physician to get laboratory results as well as other information in a timely manner.

Other changes under development include utilization of a standard report format, minimizing the amount of time required to provide a meaningful patient report. An important aspect of this is the availability of nurses simultaneously available to give and receive report. The team has developed a protocol allowing three acceptable means of transferring information from unit to unit.

The team is optimistic that by implementing these reengineering solutions the average time from decision to admit until admission will be reduced from $90 \mathrm{~min}$ to less than $45 \mathrm{~min}$. In addition, the team hopes to achieve the following: reports being delivered with one phone call, immediate medical coverage and orders available when a patient arrives on the floor, and the consistent implementation of the "bed ahead" concept. Each will provide additional capability for the ECC to handle more cases, while improving customer service, satisfaction, and outcomes.

\section{SUMMARY}

In early 1994 St. Mary's hospital made a strong commitment to CQI. A large team was formed to evaluate the whole process of the ECC, from when the patients entered to when they left. The team started to flowchart every process that occurred. Brainstorming and multi- 
voting and nominal group techniques were used to identify and address the key causes for delays in processing patients in and out of the ECC. Four key causes were identified: (a) transportation of $O B$ patients to the $O B$ floor, (b) registering patients and getting them into the ECC, (c) availability of supplies and equipment in the emergency department, and (d) the patient admitting process to the hospital from the ECC. Using the FocusPDCA approach and TQM tools, the teams were able to design processes that resulted in significant improvements. Also teams were also to improve the way emergency care was delivered by focusing on developing critical pathways for the above processes; another example of the utility of the CQI/reengineering approach. Organizational culture was changed to one of more cooperation and understanding, reflecting definitively measurable quality results.

\section{References}

1. Eubanks P. The CEO experience: TQM/CQI. Hospitals 1992; 66:24-36.

2. Gothard L, Wixson N. Charting a course for continuous quality improvement. Risk Mgmt 1994;41:27-33.

\section{American College of Medical Quality}

For information on membership and educational programs call 